\title{
ZUM ANGELSÄCHSISCHEN REIMLIED.
}

V. 5 ff. des Reimliedes sind in der handschrift folgendermassen uberliefert:

\author{
wic ofer wonzum. wennan zonzum. \\ lisse mid lonzum. leoma zetonzum.
}

Grein's text schliesst sich eng an die uberlieferung an. $\mathbf{E r}$ corrigiert wazzum mit recht in wâzzon, das bereits durch den reim auf das vorausgehende zefêzon gefordert wird, interpretiert wic als wicz, zweifelsohne ebenfalls mit recht, und setzt fur wennan ein wrennan. Das ganze gibt er dann, Germ. $\mathrm{X}, 306$, so wider: Ornati currebant equi (ornatos agitabant equos?) in campis admissariorum gressibus suaviter cum longis membrorum festinationibus. Gegen diese auffassung aber erheben sich gewichtige bedenken. Für wezan lässt sich weder die bedeutung currere noch agitare nachweisen. Die stelle Ex. 180, welche Grein im glossar II, 655 f. fur die erstere bedeutung heranzieht, kann nicht als beweis dienen, denn sie ist selbst unklar. Sodann erregt wrennan zonzum als 'admissariorum gressibus' anstoss, selbst wenn man das falsch construierte *wrenna noch ahd. reineo, rein(n)o Graff I, $978 \mathrm{f}$. in * wrêna verbessert; denn man mulsste immërhin den gen. pluralis erwarten. Endlich ist der ansatz zelonz $=$ 'motus acceleratus, festinatio' Grein I, 462 schwerlich haltbar; denn das verbum tenzan heisst ursprünglich wenigstens nicht schlechtweg 'eilen', wie Grein II, 525 annimmt, sonderu 'tendere aliquo', eigentlich 'sich nähern', wie die zahlreichen verwanten des wortes lebren. Ich glaube es ist $\mathrm{zu}$ lesen

\section{frætwe wêzon}

wicz ofer wonzum wræ̂nan zonzum

lisse mid lonzum leoma zehonzum, 
und ubersetze 'schmuck trugen die rosse auf den wangen, stolzen schrittes freudig mit langem gliederbehang', ziehe also wonzum zu wonze swn. 'wange', nicht zu wonz gefilde, und nehme wrênan als dat. pl. zu dem adj. wrêne lascivus, zu dem ja auch reineo emissarius gehört. Zur sache mag es genligen auf die eahta meáras fátedhleóre Beow. $1036 \mathrm{f}$. zu verweisen, und hervorzuheben, dass in die schilderung des reichtums und gluckes deren der dichter zu eingang sich ruhmt, besser die beschreibung der pracht seiner rosse passt, als eine angabe uber wettrennen oder dergleichen.

Auch die folgenden verse scheinen mir nicht in ordnung 2u sein. Jedenfalls enthält world onspreht $9^{\text {b }}$ auch nach der umsetzung in woruld onspreaht einen metrischen fehler, der vers ist zu kurz. Zudem ist das á $\pi$ ò xocvoṽ anstössig. Ergïuzt man aber irgend etwas vor woruld, so fehlt dem ersten stucke des satzes $b \hat{a}$ was wostmum âwoht das subject. Auch scheint die schilderung eines uppig fruchtbaren jahres, welche nach Grein's ubersetzung in den zeilen enthalten ist ('quum fuit plantis expergefacta terra frutectosa sub coelis expansa'), ebenso wenig in den zusammenhang zu passen, ale der schlusesatz, dass die 'welt' mit einer reiterschaar bedeckt gewesen sei ('turma equestri supertecta'). Ich möchte dem gegenuber glauben, dass die participia sich alle auf den dichter selbst boziehen. Man könnte also etwa denken, dass der ursprungliche text gelautet babe

pæ wæs ic wæstmum âwæht, ofer woruld onspræht, under roderum âræht, rôdmæżne oferpæht,

aber auch dieser text bietet noch grosse schwierigkeiten. Die erste halbzeile musste etwa heissen 'da war mir reichtum erwachsen', der zweite 'ich war uber die welt erhaben', dic dritte 'hier auf erden erhøht'. Aber kőnnen die worte das bedeuten? Einfacher ist der schlussvers: 'eine schaar von beratern (oder wenn man Grein's radmazne aufnimmt, von reitern) schutzte mich'. Vielleicht gelingt es einem andern, in der hier angedeuteten richtung eine befriedigende erklărung zu finden.

Zu v. 11 vermutet Grein fur zerscipe die bedeutung (ioculatio', indem er auf zear 25 und zeorran verweist (II, 442. 493). Das ist sebr bedenklich. Wollte man auch zugeben, dass 
zerscipe etwa für zearscipe (aus zearr-) stehen könnte, so mulsste doch das zear von v. 25 ausser betracht gelassen werden, denn dort verlangt der reim die form žêr, also das wort 'jahr'. Man wird sich also einstweilen begnugen mussen, zerscipe als unverständlich zu bezeichnen.

V. 13 scrifen scrâd z̧ladd ist zu kurz; man mtisste mindestens scrîfen ansetzen, was aber auch keinen sinn ergibt. Die stelle muss verdorben sein.

Fur v. 18 habe ich Beitr. IX, 235 vorgeschlagen zu lesen peznunze pêze, peódne was ic wêze. Dabei war nicht beachtet, dass der dichter selbst die stellung eines herren einnimmt. Es wird also penden einfach beizubehalten sein. Das folgende enthălt dann die weitere augfuhrung zu r. $18^{\mathrm{b}}$.

V. 21 ändert Grein das uberlieferte hyzedryht in hîzedryht 'communitas familiarium' (gl. II, 78). Das musste mindesteus hîwzedryht heissen, denn in allen deutlichen compositis bleibt das $w$ des ersten wortes, vgl. hîwcuð, hînzedal, hîwrêden, hîwscipe bei Bosworth-Toller; das einzige hîred, angl. hiorod kann hiergegen nicht in betracht kommen. Durch Grein's lesung aber wird ausserdem der glatt uberlieferte vers (hyzedryht befeóld $\dot{\sqcup} \times \dot{-} \times \mid \stackrel{\prime}{\rightarrow}$ ein $\mathrm{E}$ mit auflösung der ersten hebung) sebr belastet. Verse der form $\dot{-} \times \dot{-} \times \mid \dot{\prime}$ kommen $\mathrm{zwar}$ hie und da vor, Beitr. X, 266. 309 f., aber fast alle diese verse haben in der senkung eine silbe wie -or, -el, -um u. dgl. aus silbenbildendem $r, l, m$ etc., welche, wie Beitr. $\mathrm{X}, 480 \mathrm{f}$. gezeigt ist, öfter als unsilbisch gerechnet werden. Das gilt auch von dem verse zaldorwordum zốl Reiml. 24a, wo sicher zaldrwordum zu lesen ist. Jedenfalls wird man ohne die zwingendsten grunde solche verse nicht erst durch conjectur in den text hineinbringen durfen. Sonach ist hyzedryht einstweilen beizu-

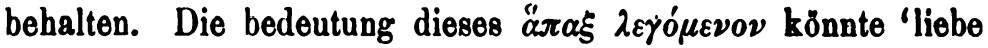
schaar' sein.

V. 23 f. liest Grein folgendermaassen:

$$
\text { swylce eorto ol ấnte ic aldorstol, }
$$

und ubersetzt Germ. X, 306 'pedisequorum potestatem habui: sicut segetis plantam (dazu die note: ôl id quod crevit, planta?) habui sedem dominicam'. Im glossar orklärt er Il, 480 stepezonzum zweifelnd als dativ eines *stepezonz gressus, oder eines 
*stepezenza pedisequus, ôl dagegen II, 321 als praeteritum zu alan. Das letztere ist gewiss allein möglich, denn wenn $\hat{o} l$ substantivum wäre, musste eorðan statt eorठe stehen. Fassen wir aber $\hat{o} l$ als verbum, so steht bei Grein's interpunktion swylce in der luft. Man muss es deshalb zum vorausgehenden ziehen: stepezonzum weóld snylce eorðe òl: ahte ic aldorstôl u. s. w. 'stolzen schrittes berschte ich über alles was die erde hervorbrachte, den herscherstuhl hatte ich inne'.

V. $24^{\text {b }}$ ist tuberliefert als zomel sibbe ne of oll. Dafur setzt Grein zomen sibbe ne of-ôl 'laetitia pacis non decrescebat', was mir wider sehr bedenklich vorkommt. Denn einerseits wird sich die intransitive bedeutung 'decrescere' fur ofalan schwerlich wahrscheinlich machen lassen, andererseits wäre die wortstellung, sibbe nach zomen, sehr ungewöhnlich. Ich glaube, man mulsste bei Grein's lesung wol sibbe als dativ, und das wort in der freilich sonst nur noch einmal, Sal. 4, bezeugten bedeutung 'sippe' fassen. Auch die dreisilbige senkung in einem verse des typus $D$ oder $E$ wäre auffällig (allerdings könnte das $e$ von ne vielleicht elidiert werden). Ich glaube also, dass auch für diesen vers noch keine heilung gefunden ist. An zomelsibb ne ofcôl wird doch schwerlich $\mathrm{zu}$ denken sein.

V. $25 \mathrm{f}$. lauten in der handschrift

ac wæs zefest zear. zellende sner.

wuniendo wær. wilbec biscær.

Dies behãlt Grein bei, nur dass er $25^{\text {b }}$ um des reimes auf zear willen snear einsetzt. Aber, wie bereits Beitr. IX, 235 angemerkt wurde, liegt es auf der hand, dass auch hier vierfacher reim anzusetzen ist, und das ist nur möglich, wenn man die reimwörter als zêr, snêr, wêr, biscer (oder biscoer) auffasst. Schon damit wird Grein's deutung von zear als 'ioculatio' (oben s. $346 \mathrm{f}$.) hinfällig. Auch seine erklärung von zefest ist falsch. Er zieht die form $\mathrm{zu}$ einem $\mathrm{adj}$. ${ }^{*} z e a f$, welches er weiterbin gl. I, $491 \mathrm{mit} z a f s p r \hat{e} c$ etc. zusammenstellt; $\delta \hat{d} f$ - aber hat aus leicht ersichtlichen grunden langes $\hat{a}$, und ahd. gebôs nugae, das Grein zum schlusse auch heranzieht, ist naturlich nichts anderes als das bekannte gi-bôsi Graff III, 217 (vgl. zum uberfluss die von Grein angezogene stelle in den Ahd. gll. II, 558,63). Ausserdem passt zefest mit kurzem vocal nicht in 
den vers, der zum typus B gebōrt. Es lăge am năchsten, zêfest zu setzen, aber diese form wăre kaum zu erklăren. Darf man an anglisches zef-fest $=$ ws. zief-foest 'begabt $=$ reich an gaben' denken? Das wort begegnet, freilich in etwas anderen sinne, Cräft. 36: wồbora ziedda ziffœst. Man kōnnte dann tubersetzen 'sondern gesegnet war das jahr, es ertönte die saite' u. s. w. Die folgende zeile verstehe ich nicht, denn an die erklärung Grein's kann ich nicht glauben.

V. 30 lautet burzsele bifade, beorht hiffade. Für hifian ist Beitr. X, 502 länge des wurzelyocals nachgewiesen, folglich muss hier im reime auch bifade gesetzt werden, zumal auch im folgenden nur langsilbige verba stehen. In ags. ist dies bîfian allerdings nur hier zu belegen (alle andern stellen in der poesie fordern oder gestatten bifian resp. biofian), es wird aber durch ahd. bîba gestitzt; vgl. thô rauard thâr sâr, urầla mihì erdbîba Otfr. V, 4, 21.

V. 31 ellen eacnade. ead beacnade ist von Grein dem sinno nach richtig in ellen eácnade, eád weacnade verbessert worden; nur ist weacnade für wacnude keine mögliche form. Vielmehr ist auch hier in anglischer form der reim als êcnade : wacnade herzustellen; $\hat{e}$ reimt hier auf $a$ wie oben wêr : bisccer 26.

V. 38. Ueber den reim frætwum : zætwrum 8. Beitr. IX, 235.

V. 40 foldan ic freotode, folcum ic leotode. So richtig Grein im text, dagegen leótode Germ. X, 425 und gl. II, 173, 'populis cantavi' Germ. X, 306. Dadurch wird der roim zerstơrt. Im altsăchsischen heisst lithôn 'fuhren': tiuchid up te stade, lidôd sie (die fische) te lande Hel. 2632, reflexiv that sie im thanan ôdran uueg erlôs fôrin, lidôdin sie te lande Hel. 684, und so ist ags. liðian, leotian auch nichts als 'fuhren', nur dass es an unserer stelle intransitiv gebraucht wird: 'dem volke war ich fuhrer'. Dazu passen auch die composita aliđian 'entfubren' und tôlið̇ian = mhd. zeviieren: hê paet andweorc of Âdames | lice aleoðode ond him listum ateáh | rib of sidan Gen. 176 ff., bið bat heáfod tôhliden, handa tôliðode Seelen 109. Wegen Guthl. 362 f., wo Grein ebenfalls ledoian ansetzte, vgl. Beitr. X, 517.

V. 42. Grein's deutung dieses verses ist 'höchst bedenklich, denn zetonze und zehonze können doch nicht starke nominative von adjectivis sein, wie er annimmt (fur zetenze und 
zehenze), und wie soll man schwache flexion rechtfertigen? Auch teala scheint mir weder gl. I, 408, wo es als gen. pl. von til n. gefasst wird, noch II, 521, wo es zu talu gestellt wird, befriedigend erklärt zu sein.

V. 43 f. sind die reimwörter in der anglischen form hrêh : scêh : nêh : flêh herzustellen, Beitr. IX, 235. Keine verbesserung des handschriftlichen zenîtè nihtes in fleáh $49^{\mathrm{b}}$ ist Grein's conjectur zewitod nihtes infleáh, denn einerseits existiert ein zewitod $\mathrm{m}$. 'die einem bestimmte lebenslage' nicht und lässt sich auch aus dem part.-adj. witod nicht so ohne weiteres ableiten, andernteils verstōsst die änderung gegen die regel, welche alliteration des nomens resp. ersten nomens einer halbzeile verlangt: eine regel die jedenfalls beim conjecturieren zu beachten ist, wenn auch hie und da ausnahmen vorkommen, wie im Reimlied selbst balavum hêr zelloten 79 mit alliteration auf $h$. Der handschriftliche text kann wol beibehalten werden, wenn man fleáh resp. flêh als 'flucht' fasst: 'der muss jetzt nächtlicher weile fliehen, der ehedem am tage in ehren stand'.

Schwierig ist wider v. 45. Ueberliefert sind die reimwörter in der form dyre: feor. In einem anglischen gedicht können diese entweder deore : feóre oder deór:feor gelautet haben, aber nicht dŷre : fôre wie Grein schreibt, Beitr. IX, 235. Wenn deóp in v. 45 richtig ist, muss das endwort des verses zweisilbig sein (typus C); wir kămen also zu dem reime deore : feóre. Dies feóre gleich fyre zu setzen, also dem ags. eine dem ahd. alts. fiur entsprechende nebenform feor zuzuschreiben, wird schwerlich angehn, da diese form sonst nirgends vorkommt, wenigstens aus dem compositum feórstudu, -stuðu (destina feurstud ${ }^{1}$ ) in den alten glossen am schlusso des.hymnus Cædmons im Ely MS., obstipum feorstupu in den Cleopatraglossen, Wright-Wulcker I, 280, 10. 461,3] wol kaum entnommen werden kann, da dieses wort 'querbalken, stlitzbalken' bedeutet. Es bliebe also wol nur feóre als dativ von feorh ubrig. Dies könnte dem dat. breóstum 46 parallel stehen,

1) Man beachte dass hier stud noch ohne das $u$ erscheint, welches die kurzsilbigen consonantischen feminina sonst nach dem muster der a-stämme angenommen baben, Beitr. IX, 249. 
nur fragt es sich, ob feóre zu ferh eine correcte anglische form ist. Nach dem was Zeuner, Die sprache des kent. psalters s. $85 \mathrm{f}$. uber die flexion von jweorh und feolan im Ps. bemerkt hat, wulde man eher fere oder fêre zu erwarten haben, vgl. namentiich den conj. fele zu feolan, aus *felhe, nicht *feolhe. ${ }^{1}$ ) Andererseits weicht aber der Ps. wider von der gewöhnlichen behandlung der gruppen $l h$ und $r h$ ab, indem tweoru nom. sg. f. zu Jwerh küze des wurzelvocals trotz dem ausfall des $h$ voraussetzt, gegen Beitr. X, $487 \mathrm{ff}$. Die ganze frage bedarf noch einer genaueren untersuchung.

V. 48 lauten nach der lesung von Thorpe und Schipper môdes zecynde zrêteð̀ unzrynde znorn efen pynde.

Fur das letzte wort vermutet Grein II, 362 zweifelnd die bedeutung 'cisterna, lacus', und demgemăss tubersetzt er Germ. $\mathrm{X}, 306$ 'animi naturam aggreditur fundo carens moeror cisternae instar'. Dagegen ist zu erinnern, dass zwar ein abgeleitetes verbum pyndan existiert, aber kein substantivum pynde, soviel bekannt ist, sondern nur pund (= neuengl. pound) in ags. pundfald pferch (of Jam putte on hacan pundfald, of hacan pundfalde of eft on taet eferfearn in einer urkunde des königs Eadgar vom jahre 961 bei Kemble, Cod. dipl. VI, 41) und pundbreche LL. Henr. 1, 40 (weiteres s. bei Skeat, Et. Dict. unter pound ${ }^{2}$ und pinfold). Wie pund eine 'einschliessung' uberhaupt bedeutet, so heisst auch pyndan allgemein 'einschliessen' und dadurch 'zurlickdrăngen', so in der einzigen stelle wo das wort in der poesie begegnet: pat is t̂tun scyld eal forpynded, warzठu aworpen Crist $97 \mathrm{f}$. Speciell aber bodeutet es 'eindämmen', 'aufstauen', wie sich in den folgenden stellen der Cura pastoralis zeigt: Jat unzeornfulle môd ond

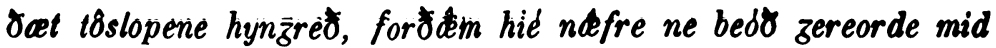
zôdum weorcum, ne hie nellað hie zehceftan ond zepyndan hiora môd, swylce mon deópne pool zewerize, ac hê lât his môd tôflôwan on tat ofdele ziémeliéste etc. 283,11 ff., und ac tat mennisce môd haef watres Jeáw. Đat water, ठonne hit bið

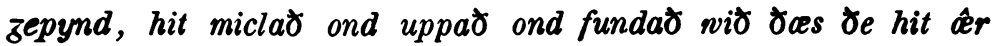
from côm, ठonne hit flôwan ne môt ठider hit wolde. Ac zif sio

1) Vgl. auch den acc. snere ans *snearhe Wyrde 82. 
pyndinz wierठ onpennad otঠe sió wering wirठ tôbrocen, ठonne tôflêwo hit eall 277,5 ff. Demnach galt pund ohne zweifel auch einmal in dem sinne von 'damm, wehr' oder 'schleuse', und hieran bei unserer stelle anzuknupfen, liegt, meine ich, ziemlich nahe. Ein adj. efenpynde gibt $\mathrm{zwar}$ auch noch keinen rechten sinn, wol aber dürte ein mit geringer anderung daraus herzustellendes oferpynde ${ }^{1}$ ) billigen ansprtuchen genugen. Der schmerz, zrorn, wird dem aufgestauten gewăsser verglichen, das den damm tibersteigt oder bricht, und wie es weiter in $\nabla .50^{b}$ heisst (nachdem in $50^{\mathrm{a}}$ ein neues bild eingeschoben ist, bealof $u$ s brinneঠ), bittre tôrinneð, d. h. gewaltsam und vernichtend sich nach allen seiten ergiesst.2)

Fur v. 50 ist eben nur anzumerken, dass statt des uberlieferten byrneð : tôyrneð des vierfachen reimes wegen brinneð : tôrinneঠ herzustellen ist, oder vielleicht genauer gleich brinnið : tôrinnið, denn die folgenden sinnip, cinnið, blinnið können doch wol nur tberbleibsel der ursprunglich durch das ganze gedicht sich erstreckenden altertumlichen orthographie sein.

Damit ist zugleich schon angedeutet, dass ich Grein's auffassung von sinnip 52 als sin-nî̀ nicht billigen kann. Ein reim wio șin-niðठ : cinnið wäre für unser gedicht ganz unerhört, Sinnið muss eine verbalform sein, wie die tubrigen reimwörter, und darauf weist auch die uberlieferung hin, welche nicht sârne, sondern sâr ne hat. Freilich, wenn man mit Grein Il, 453 für sinnan die bedeutung 'reputare, curare, rationem habere alicuius' annimmt, bleibt die stelle dunkel. Aber jene bedeutung ist durchaus unursprunglich. Sinnan bedeutet ahd., wie man bei Graff VI, 227 ff. ersehen kann, zunăchst 'gehen', dann 'streben'. Die letztere bedeutung haben wir ags. im Guthlac: ne ic mê eorơvelan ôwiht sinne ne mê mid môde micles zyrne $290 \mathrm{f}$. Mit der grundbedeutung 'einhergehen' kommt mau Gen. 1851 f. aus, wenn man nur 1852 pat für ond setzt: Abraham ist mit Sarah nach Egyplen gekommen, und alle preisen Sarahs schönheit:

1) Congbeare gibt als lesung der hs. ofen pynde. Aber da Thorpe und Schipper in der lesnng efen libereinstimmen, kann ich darauf kein gewicht legen.

2) Grein's erklärung von to-yrneð als 'accurrit' ist natürlich zu verwerfen. Auch das metrum spricht dagegen. 


hié pæet cừ dydon
heora folcfrêzan, pæt fæzerro lŷt
for æðelinze idese sunnon,
ac hié Sarrâan swî̉or micle
wynsumne wlite wordum hertedon

'und sie verklindeten ihrem herrn, dass wenige der frauen, die vor dem edeling wandelten, an schönheit sie uberträfen' u. s. w. Dann bleibt noch eine stelle im Andreas ubrig, v. 1279. Andreas wird den ganzen tag lang gepeinigt, und

\section{hrâ weorces ne sann,}

wundum wêriz. pầ côm wôpes hrinz

purh pæes beornes breóst blât ât faran

u. s. w. 'Streben' passt hier gar nicht. Im glossar versieht Grein die stelle einfach mit einem fragezeichen, in den Dichtungen II, 35 ubersetzt er 'die leiden fühlte kaum noch der wundenmatte leib'. Dagegen ist einzuwenden, dass 'fuhlen' aus dem grundbegriff von sinnan nicht abzuleiten ist, und dass der folgende klagruf schlecht sich anschliesst, wenn Andreas schon bis zur bewusstlosigkeit gepeinigt worden ist. Auch scheint mir der ganze gedanke etwas allzu modern. Ich nehmo vielmehr an, dass das ursprüngliche sinnan 'gehen' sich in die beiden bedeutungen 'hingehen, streben' und 'fortgehen, vergehen, aufhören' gespalten hat, wio etwa mhd. sich bevegen, ubersetze also etwa dem sinne nach 'kein aufhören, keine unterbrechung des leids kam dem wundenmuden leibe.' Im Reimlied bedeutet dann sâr ne sinniठ einfach 'sein schmerz hört nicht auf'.

Unklar bleibt auch dann noch die zweite vershălfte, sorzum cinnið. Im Sal. 107 begegnet ein auch noch unerklärtes forcinnan etwa in dem sinne von 'tiberwăltigen, ergreifen', Läge dasselbe verbum hier vor, so mlisste sâr noch subject sein, was mir sehr bedenklich vorkommt. Bis eine bessere lösung gefunden wird, möchte ich fragen, ob man nicht cinnan als (neugebildetes?) intransitivum zu cennan 'zeugen, gebären' fassen darf, mit der bedeutung etwa von 'wachsen'. Also 'er nimmt zu an sorgen'?

Aus den folgenden zeilen, die ich zum teil auch nicht verstehe, hebe ich nur noch hervor die verse $70 \mathrm{ff}$. Dieselben lauten bei Grein: 
mê pæt wyrd zewæf and zewyrht forzeaf pæt ic zrôfe zræf and pæt zrimme zeræf fleón flâsce ne mæz.

Dass in dem ersten verse forzaf zu lesen ist, habe ich Beitr. IX, 235 bemerkt. Ausserdem nehme ich anstoss an Grein's conjectur zercef für handschriftliches zrref. Das wort ist unerklärt, Grein selbst kann nur zweifelnd als bedeutung desselben 'constitutum, destinatum' vermuten. Ohne zweifel ist zrof in $71^{\mathrm{b}}$ nur widerholung des $z$ ref von $71^{\mathrm{a}}$, man braucht sich also nicht zu ängstlich an seine buchstaben $\mathrm{zu}$ halten. Mit scraf wird dem verse wie dem sinne vollkommen gentigt. TÜBINGEN, 18. august 1885 .

\section{DIE HEIMAT DES BEOWULFDICHTERS.}

In diesen Beiträgen XI, $173 \mathrm{ff}$. sucht $\mathrm{G}$. Sarrazin seine annahme eines nordischen ur-Beowulf in liedform unter anderem auch durch eine reihe von sprachlichen argumenten zu stlutzen. Ich möchte diesen versuch nicht obne einsprache bingehen lassen; es könnte sonst doch am ende jemand auf den wahn verfallen, als verhielte es sich mit den besprochenen dingen wirklich so wie der verfasser jenes aufsatzes seine leser glauben machen will. Dabei lebne ich es ausdrucklich ab, auf discutable punkte einzugehen, d. h. auf dinge, wo meinung gegen meinung stehen kann. Meine kritik soll sich bloss auf dinge erstrecken, welche Sarrazin als angeblich factische grundlagen seiner auffassungen benutzt. Vollständigkeit in der widerlegung des widerlegbaren habe ich dabei nicht im auge.

Seine beweisfuhrung hebt Sarrazin s. 173 mit der behauptung an, der Beowulf wie die tubrige ags. dichtung zeige altnordischen einfluss im wortschatz und in der phraseologie. Diese behauptung wird dann weiter gestutzt auf eine liste von 36 wörtern, die dem Beokulf entnommen sind, aber auch sonst in der ags. poesie begegnen, dagegren der ags. prosa fehlen. Diese 36 poetischen wörter werden danach fur scandinavische fremdwörter erklärt. Sehen wir uns dieselben also etwas näher an! 